\title{
Vegetation change detection research of Dunhuang city based on GF-1 data
}

\author{
Zhaopeng Zhang ${ }^{1,2} \cdot$ Zengyuan $\mathrm{Li}^{1,2} \cdot \mathrm{Xin}^{\operatorname{Tian}^{2}}$
}

Received: 18 May 2017/Revised: 31 August 2017/ Accepted: 18 January 2018/Published online: 30 January 2018

(C) The Author(s) 2018. This article is an open access publication

\begin{abstract}
This study selected the Dunhuang city with the unique landscape pattern, its oasis surround by desert, for studying the vegetation change. Based on two period GaoFen-1 images, combined with field survey data, the dimidiate pixel model and fractional vegetation coverage estimation model were applied to detect the changes of local vegetation coverage from July 2013 to July 2015. Analysis showed that during the study years, the Grain for Green project had a remarkable effect on the changes. The vegetation types of intermediate high and very low grades increased by 0.66 and $6.78 \mathrm{~km}^{2}$ respectively. The low vegetation coverage decreased by $23.87 \mathrm{~km}^{2}$. The vegetation coverage types of intermediate grade increased by $88.97 \mathrm{~km}^{2}$ because of the planted forest, which accounted for $10.84 \%$ of the study area. The vegetation coverage types of high grade reduced by $72.47 \mathrm{~km}^{2}$. This change effectively prevents the spread of desert and lays a good foundation for the ecological construction of Dunhuang city.
\end{abstract}

Keywords Dunhuang city · GF-1 - Vegetation coverage · Grain for green

\section{Introduction}

Oasis refers to a green land with weeds in the desert. Dunhuang oasis, is one of the main bases for human settlement and production in the arid and semi-arid regions of Northwest China. Fractional Vegetation Cover (FVC) is the basic index of ecological system, which is affected by the changes of natural environment and human activities. In order to understand the current situation, trends, driving forces, and environmental impacts of the vegetation cover in desert oasis, using remote sensing date, numerous studies have been performed (Yang et al. 2010; Liu et al. 2013; Zhang et al. 2015). However, there are few research

Xin Tian

tianxin@caf.ac.cn

1 Surveying and Mapping Institute of Science and Technology, Xi' an University of Technology, Xi' an 710054, Shanxi, China

2 Institute of Forest Resources Information Techniques, Chinese Academy of Forestry, Beijing 100091, China based on the Chinese high resolution satellite constellation. Based on two period GaoFen-1 (GF-1) images, the dimidiate pixel model and vegetation coverage estimation model were applied to detect the changes of vegetation covers from 2013 to 2015 in Dunhuang oasis. The goal is of this study is to provide a reference for accurately understanding the situation of regional ecological change and evaluating the effectiveness of the ecological projects.

\section{Materials}

\subsection{Study area and data}

Dunhuang city of the northwest of Gansu province, is located at the ancient Silk Road. The territory is surrounded by mountains at three aspects. The Danghe river is the only water source for the survival of the city. GF-1 images (two for July 2013 and two for July 2015) covering Dunhuang city were obtained from China Center for Resources Satellite Data and Application. GF-1 is a kind of high resolution earth observation satellite with independent 
property rights in China (Fig. 1). Data pre-processing was conducted by the ENVI software (version 5.4.1).

\subsection{Image data preprocessing}

The RPC file of GF-1 images in 2013 and the corresponding DEM from Geospatial Data Cloud were used to make the geographic correction. After that, the GF-1 images in 2015 were processed to match with the GF-1 images from 2013 by means of an image-to-image matching method provided by the ENVI Imagine software. The root mean square (RMS) control of each control point is less than 1 pixels, and the total root mean square error (RMSE) is 0.49 (Fig. 2).

\section{Methods}

\subsection{NDVI and vegetation coverage}

The normalized difference vegetation index is known to be an index of vegetation conditions (Carlson and Ripley 1997). This index is expressed as:

$\mathrm{NDVI}=(\mathrm{NIR}-\mathrm{R}) /(\mathrm{NIR}-\mathrm{R})$

NIR and R represent the reflectivity of the near infrared and red bands, respectively (Ye et al. 2009).

Combined with the content of the study, the NDVI gray scale images of different time images are obtained by using NDVI. It is necessary to transform the vegetation index into the degree of coverage. In a certain extent, FVC can make up for the disadvantage of NDVI, in which NDVI is difficult to distinguish the low coverage vegetation and the coverage is easy to be saturated. On the contrary, so as to effectively stretch the range of vegetation information. The vegetation cover can be divided into two types, pure and mixed pixels by the different structure characteristics. A mixed pixel includes two kinds of information of soil and vegetation. Assume that the vegetation index is NDVIveg, and the vegetation index is NDVIsoil, and the mixed pixel vegetation index is:

NDVI $=$ FVC $\times$ NDVIveg $+(1-$ FVC $) \times$ NDVIsoil

The vegetation coverage model is:

$\mathrm{FVC}=($ NDVI - NDVIveg $) /($ NDVIsoil - NDVIveg $)$

In the above formula, NDVIveg represents the normalized difference vegetation index of the whole vegetation cover, NDVIsoil represents the normalized difference vegetation index of bare soil.
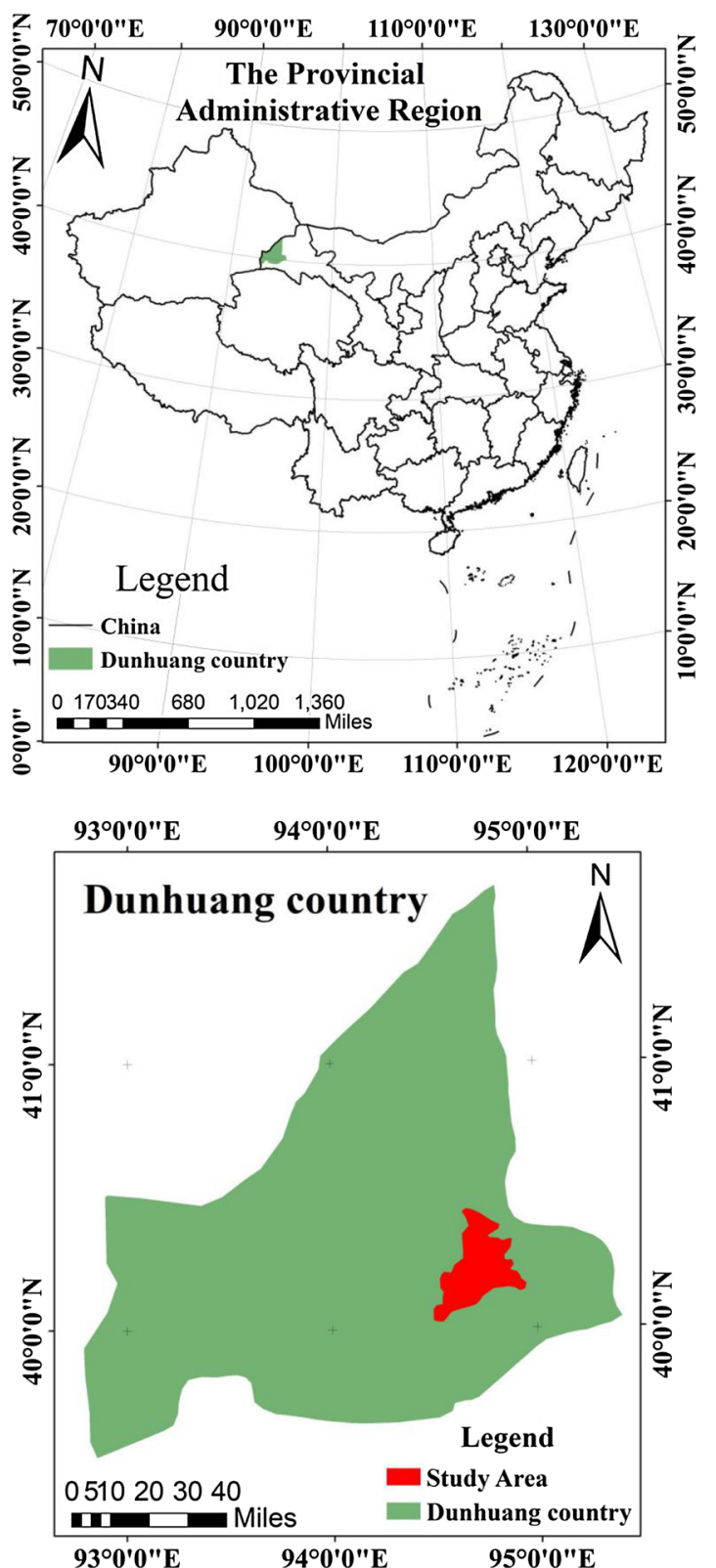

Fig. 1 Geographical position of study area

The key of the mixed pixel decomposition method is the acquisition of NDVIveg and NDVIsoil. In this paper, NDVIsoil and NDVIveg parameters were generated by using the NDVI threshold of land cover type. The following expressions are used to calculate the NDVIsoil and NDVIveg:

$$
\begin{aligned}
\text { NDVIsoil }= & b 1 * n 1+b 2 * n 2+b 3 * n 3+b 4 * n 4 \\
& +b 5 * n 5 \\
\text { NDVIveg }= & b 1 * n 6+b 2 * n 7+b 3 * n 8+b 4 * n 9 \\
& +b 5 * n 10
\end{aligned}
$$



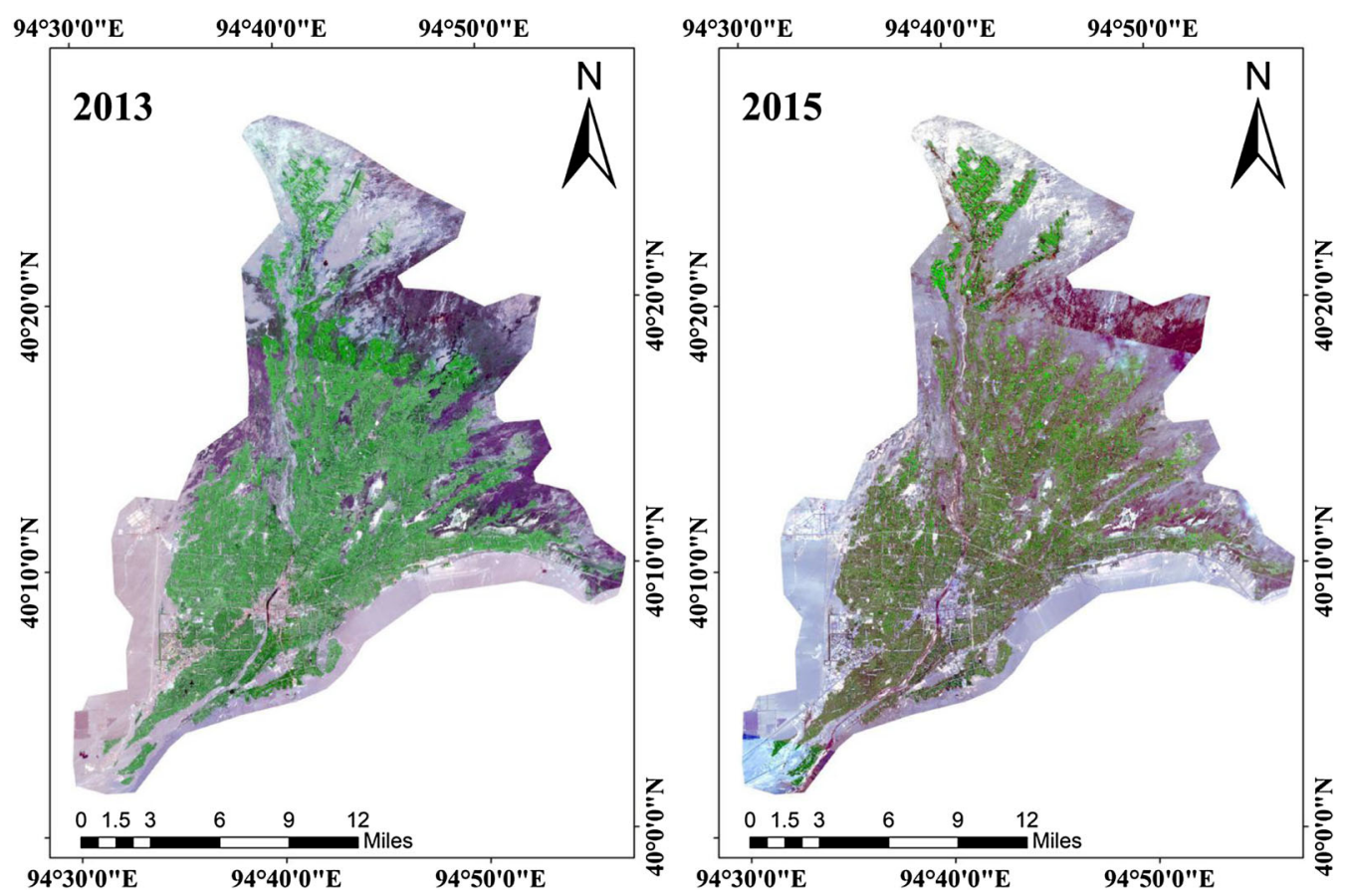

Fig. 2 GF-1 images of the study area (Left: 2013a, Right: 2015a)

Table 1 Classification scheme used in this study

\begin{tabular}{lll}
\hline Classification & Ranges & Instruction \\
\hline $\begin{array}{l}\text { Very low coverage vegetation } \\
\text { Low coverage vegetation }\end{array}$ & $0.00001-0.1$ & $\begin{array}{l}\text { Intensity of desertified land, bare soil, ground flowing sand, gobi, water bodies, etc. } \\
\text { Sparse forest land, semi fixed sandy land, low yield grassland and seriously degraded land, } \\
\text { etc. }\end{array}$ \\
$\begin{array}{lll}\text { Intermediate coverage vegetation } \\
\begin{array}{l}\text { Middle and high coverage } \\
\text { vegetation }\end{array}\end{array}$ & $0.3-0.5$ & $\begin{array}{l}\text { Fixed sandy land, light sandy land, low density forest land and cultivated land, etc. } \\
\text { High coverage vegetation }\end{array}$ \\
\hline
\end{tabular}

where $b 1, b 2, b 3, b 4$ and $b 5$, are forest land cover document, agricultural land cover document, urban land use mask files, water Mask documents and other land use mask file respectively; $n 1$ and $n 6, n 2$ and $n 7, n 3$ and $n 8, n 4$ and $n 9, n 5$ and $n 10$, are the NDVI minimum and maximum value of corresponding each mask file respectively expressed. The Eqs. 2-3 were applied to conduct the transformation of gray scale map of vegetation cover (Zhang et al. 2017).

\subsection{Classification of vegetation coverage}

According to the criteria of classification in desertification survey of China, and the characteristics of Dunhuang oasis vegetations, the coverage vegetation in the study area were divided into five grades, (1) very low coverage vegetation,
FVC from 0.00001 to 0.1 ; (2) low coverage of vegetation, FVC from 0.1 to 0.3 ; (3) Intermediate coverage of vegetation, FVC from 0.3 to 0.5 ; (4) middle and high coverage vegetation, FVC from 0.5 to 0.7 and (5) high coverage of vegetation, FVC larger than 0.7 . The detailed classification schemes are shown in Table 1. Normally, the transformation of the low level vegetation to the high level is called the vegetation growth, and the transformation of the higher vegetation to the lower one is named as the vegetation degradation. According to the above classification criteria, the vegetation coverage map of the different periods is obtained (Fig. 3). Through field survey and land use map can know vegetation classification results is right. Then, the vegetation area and its changes from 2013 to 2015 were obtained by difference operation. 


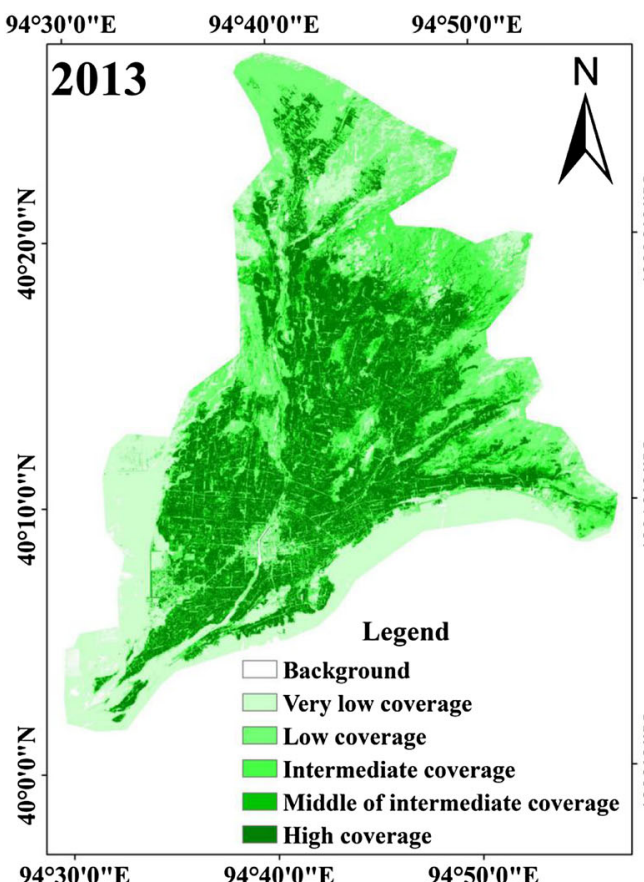

Fig. 3 The FVC classification figures (Left: 2013a, Right: 2015a)

\section{Dynamic analysis of vegetation cover}

\subsection{Overall change of vegetation coverage}

From 2013 to 2015, the vegetation coverage of Dunhuang oasis was provided in Table 2 that showed a great changes among FVC to the sustainable development of the Oasis Economy. In these five levels of vegetation cover types, compared with the situation in 2013, the coverage of vegetation increased $10.84 \%$, very low vegetation coverage and high coverage vegetation remained almost unchanged, only increased by $0.83 \%$ and $0.08 \%$ respectively, and the low vegetation coverage and high vegetation coverage were reduced by $2.92 \%, 8.83 \%$.

\subsection{The mutual changes of different vegetation cover types}

As shown in Tables 3 and 4, it is obvious that very low coverage vegetation had $93.32 \%$ positive transfer, the total change area accounted for $78.22 \%$ of the total vegetation area of the initial state. Low coverage vegetation had

Table 2 The percentage of various types of vegetation to the total area $(\%)$

\begin{tabular}{lllllr}
\hline Year & Very low & Low & Intermediate & Middle and high & High \\
\hline 2013 & 1.05 & 50.95 & 15.26 & 18.73 & 14.01 \\
2015 & 1.88 & 48.03 & 26.10 & 18.81 & 5.18 \\
\hline
\end{tabular}

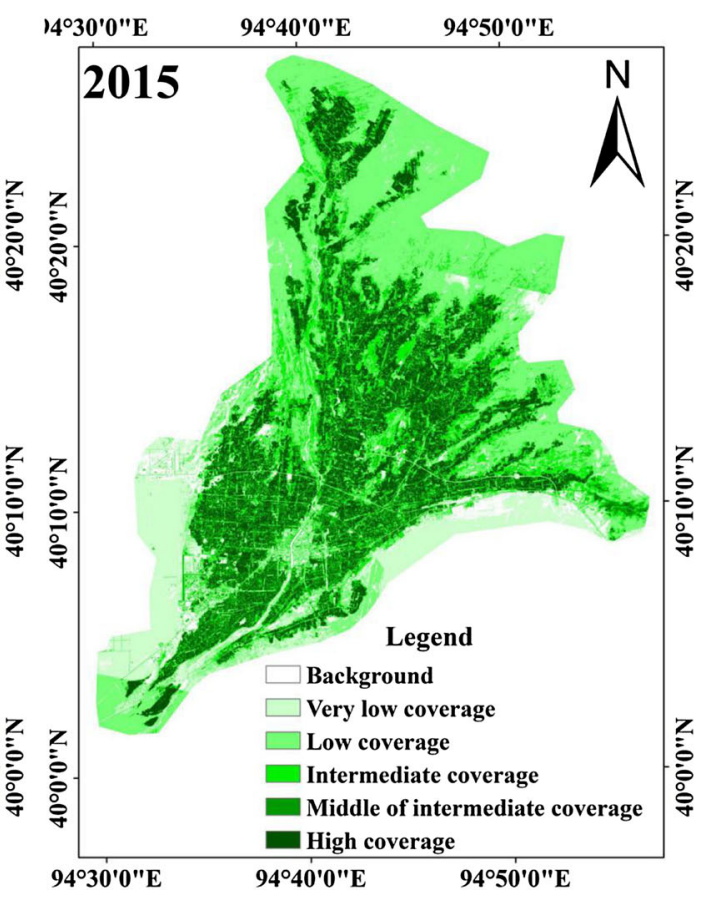

$15.64 \%$ of the transfer. In the vegetation cover degree, there were $33.20 \%$ transfer, in which the negative transfer to $15.91 \%$, positive transfer to $17.29 \%$, but its total area increased by $88.97 \mathrm{~km}^{2}$. In the middle and high vegetation coverage, there were $46.47 \%$ negative transfer and $8.40 \%$ positive transfer. High coverage vegetation transfer is relatively large, there were $79.65 \%$ occurrence of negative transfer.

\section{Analysis on the factors of vegetation cover change}

\subsection{Natural factors}

Natural factors were mainly analyzed from two aspects, namely, precipitation and vegetation.

Dunhuang city is characterized by little precipitation but large evaporation. The average amount of evaporation is 60 times of precipitation, and there almost no conversion among precipitation, soil surface water and ground water. Precipitation is one of the most important factors affecting vegetation coverage. The precipitation from 2013 to 2015 in Dunhuang city is shown in Table 5, which showed that both the annual precipitation and the summer precipitation in 2013 and 2015 were very small. As a result, the water supply for agriculture and forestry mainly came from groundwater. Water stress is the mainly problem of Dunhuang city and because of the different farming conditions, the local areas change greatly from 2013 to 2015 . 
Table 3 Various types of vegetation changes in circumstances $\left(\mathrm{km}^{2}\right)$

\begin{tabular}{llllllr}
\hline Year & Background & Very low & Low & Intermediate & Middle and high & High \\
\hline 2013 & 1002.8554 & 15.441676 & 394.1752 & 214.159766 & 154.350288 & 42.4747 \\
2015 & 1002.9178 & 8.664360 & 418.046336 & 125.188544 & 153.692192 & 114.947648 \\
\hline
\end{tabular}

Table 4 Transform situation matrix of vegetation (\%)

\begin{tabular}{|c|c|c|c|c|c|c|}
\hline 2013-2015 & $0-0.00001$ & Very low & Low & Intermediate & Middle and high & High \\
\hline Background & 0.000 & 0.000 & 0.000 & 0.000 & 0.000 & 0.000 \\
\hline $0-0.00001$ & 99.927 & 0.877 & 0.119 & 0.045 & 0.018 & 0.014 \\
\hline Very low & 0.011 & 5.804 & 3.487 & 0.118 & 0.043 & 0.032 \\
\hline Low & 0.054 & 92.080 & 84.356 & 15.749 & 5.566 & 4.123 \\
\hline Intermediate & 0.008 & 1.152 & 10.997 & 66.802 & 40.863 & 18.770 \\
\hline Middle and high & 0.000 & 0.071 & 0.778 & 13.242 & 45.110 & 56.706 \\
\hline High & 0.000 & 0.017 & 0.264 & 4.043 & 8.400 & 20.355 \\
\hline
\end{tabular}

*0-0.00001 is a special treatment for the classification, in order to remove background values and not allow them to participate in the classification

Table 5 Statistical table for precipitation information

\begin{tabular}{|c|c|c|c|c|c|}
\hline Year & Time ranges & $\begin{array}{l}\text { Precipitation } \\
(\mathrm{mm})\end{array}$ & $\begin{array}{l}\text { The days of } \\
\text { precipitation (d) }\end{array}$ & $\begin{array}{l}\text { Observatio } \\
\text { times }(d)\end{array}$ & Data sources \\
\hline \multirow[t]{2}{*}{2013} & $\begin{array}{l}\text { From January 1st to December } \\
\text { 31st }\end{array}$ & 48 & 43 & 1458 & $\begin{array}{l}\text { The data set is provided by the RP5 } \\
\text { website }\end{array}$ \\
\hline & From May 1st to July 31st & 32 & 24 & 368 & \\
\hline 2014 & $\begin{array}{l}\text { From January 1st to December } \\
\text { 31st }\end{array}$ & 58 & 37 & 1460 & \\
\hline \multirow[t]{2}{*}{2015} & $\begin{array}{l}\text { From January 1st to December } \\
31 \text { st }\end{array}$ & 33 & 42 & 1459 & \\
\hline & From May 1st to July 31st & 20 & 22 & 368 & \\
\hline
\end{tabular}

Grassland and desert are the main vegetation types of the Dunhuang city. The main types of plants are Populus euphratica, Tamarisk and Manaplant Alhagi, which can resistant to drought, wind and sand and adapt to extreme drought climate conditions. In particular, the oasis fringe area, its vegetation types are more barren, and the vegetation species structure is simple with extremely low FVC. Which is consistent with that shown in Fig. 3, the oasis surrounding is mainly the extremely low vegetation coverage.

\subsection{Social factors}

In combination with various factors, social factors were also main factors affecting the vegetation cover change in Dunhuang oasis. Social factors were mainly analyzed from these aspects, namely, population, economic, government industrial structure conduction and so on.
As the artificial forests increase, the effects of Grain for Green project are significant. According to the information from official website of the Forestry Bureau of the Dunhuang City. In recent years, the amount of afforestation in Dunhuang is 7.12 million hectares, 14.5 million hectares of afforestation from the project 0 , and 160 million acres natural vegetation were protected and managed. Only in 2015, the afforestation area is beyond 453 hectares, 1180 hectares of economic forests being newly planted, and $91.2 \mathrm{~km}$ of farmland shelterbelts were built and improved. As a result of large amounts of farmlands were converted to artificial forests, the medium vegetation coverage increased largely and the high vegetation coverage dereased.

Dunhuang was named as national comprehensive demonstration area of desertification prevention and control in 2013. 190 hectares sands were got under control in Mingsha Mountain Montenegro, Mogao Grottoes and other 

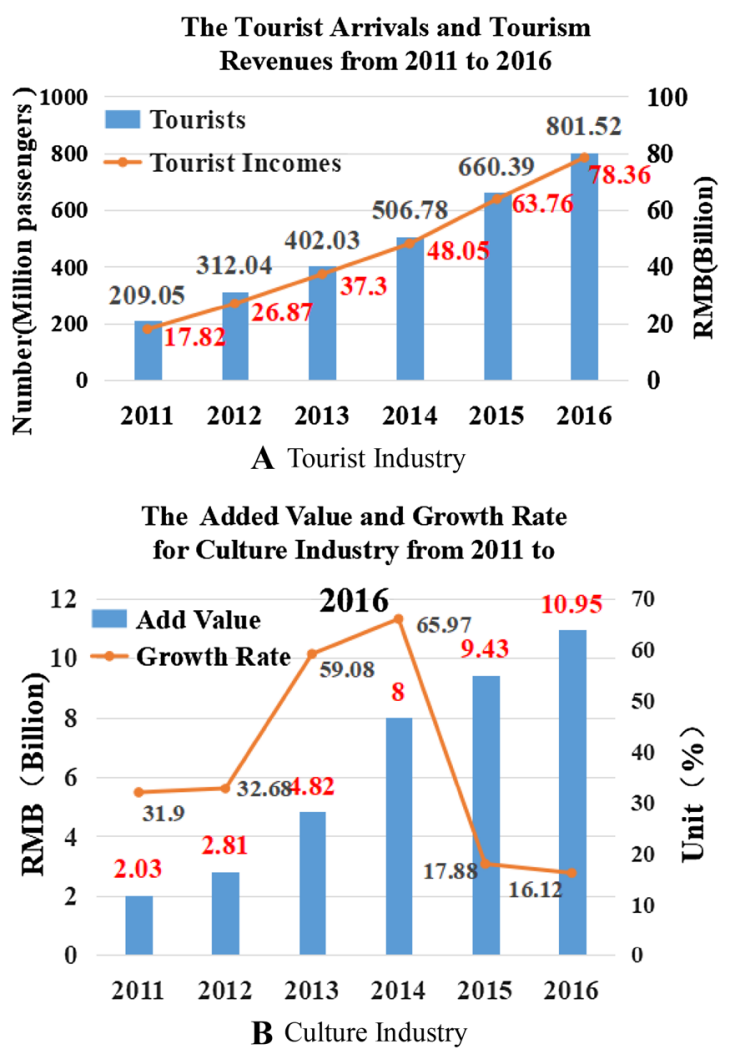

Fig. 4 Two types of economic changes from 2011 to 2016 (Top: tourist industry, Bottom: culture industry)

areas. The total area of the ban on desertification is 40,612 hectares (This detailed statistics comes from the Dunhuang City Propaganda Department). This government behavior leads to a decrease of low vegetation coverage and the increase of intermediate vegetation coverage in Mingsha Mountain, located in southeast of the study area.

With the rapid development of economy, the local industrial structure has been constantly adjusted. The Dunhuang City is an indispensable part for the "Silk Road
Economic Zone" of "the Belt and Road" (B\&R). As a result, "the Belt and Road" strategy not only brought more opportunities for the development, but also promoted its industrial restructuring, especially for the service industry. With the optimization and upgrading of industrial structure, the service industry, which is dominated by tourism, has became to be the main force for city's economic development. The income from the tourism and cultural industries is shown in Fig. 4.

During the period of the China 12th Five-Year Plan, which is scheduled from 2011 to 2015, the three industries of the city were fully developed, with the primary, second and service industries increasing by 3.7, 18.1 and $15.5 \%$, respectively. These three industrial structures tend to be optimized from 21.2: 26.5: 52.3 in the end of the 11th FiveYear Plan (2010) to 16.2: 30.3: 53.5 in the middle of the 12th Five-Year Plan (2013) and then to 13.3: 28.3: 58.4 at the end of the 12th Five-Year Plan (2015). The proportion of the first and secondary industry decreased by $2.9 \%$ and $2.0 \%$, the proportion of the tertiary industry increased by $4.9 \%$. The city's three industrial structures were shown in Table 6.

With a slight increase of the local population, the employment structure was gradually optimized. The census data published by Dunhuang City Government Network showed that by the end of 2012 and the end of 2016, the number of household population is 141,969 and 143,800 , respectively. However, with the gradual optimization of the employment structure, the labor force has been transferred from the first and second industries to the tertiary industry, and as a result, some of them moved from rural to urban areas. According to the 12th Five-Year Plan Economic and Social Development Review for Dunhuang city, published by the Dunhuang government network, the employment structure of the three industries in this city changed from 41.8:15.4:42.8 in 2010 to $37.8: 14.9: 47.3$ in 2015. The reduction of labor force in the primary industry has a direct impact on the reduction of cultivated land area. This is also

Table 6 The proportion of industry of Dunhuang city (\%)

\begin{tabular}{llllll}
\hline Year & $\begin{array}{l}\text { Primary } \\
\text { industry }\end{array}$ & $\begin{array}{l}\text { Second } \\
\text { industry }\end{array}$ & $\begin{array}{l}\text { Service } \\
\text { industry }\end{array}$ & Total & Notes \\
\hline 2006 & 27.6 & 19.1 & 53.3 & 100 & The industry status of Dunhuang city were obtained by the Gansu provincial bureau of \\
2010 & 21.2 & 26.5 & 52.3 & 100 & statistics and the Dunhuang municipal government network \\
2011 & 19.5 & 28.7 & 51.8 & 100 & The 12th Five-Year Plan: from 2011 to 2015 \\
2012 & 17.8 & 32.4 & 49.8 & 100 & The 11th Five-Year Plan: from 2006 to 2010 \\
2013 & 16.2 & 30.3 & 53.5 & 100 & \\
2014 & 14.8 & 29.1 & 56.1 & 100 & \\
2015 & 13.3 & 28.3 & 58.4 & 100 & \\
2016 & 13.7 & 25.4 & 60.9 & 100 & \\
\hline
\end{tabular}


an imply for the increase of intermediate vegetation coverage and the decrease of high vegetation coverage in Table 2.

\section{Conclusions}

In this study, based on GF-1 NDVI information and dimidiate pixel model, changes of vegetation coverage between 2013 and 2015 in the Dunhuang oasis were analyzed. The results showed that the implement of Grain for Green project has largely affected the changes. According to the changing static information, the middle and high vegetation coverage, and extremely low vegetation coverage increased 0.66 and $6.78 \mathrm{~km}^{2}$, respectively, and low vegetation coverage reduced by $23.87 \mathrm{~km}^{2}$, and the intermediate vegetation coverage increased by $88.97 \mathrm{~km}^{2}$, and high vegetation coverage area decreased by $72.47 \mathrm{~km}^{2}$.

In summary, the social factors are the most significant factors affecting the changes of the Dunhuang. In the light of the present vegetation coverage situation of the Dunhuang oasis, the protection work for the oasis should be continually conducted in order to keep the oasis stability. Meanwhile, the local water saving agriculture and Grain for Green should also be further developed.

Acknowledgements This study was supported by the Fundamental Research Funds for the Central Non-profit Research Institution of
Chinese Academy of Forestry (CAFYBB2017QC005) and the National Natural Science Foundation of China (21-Y20A06-9001-17/ 18).

Open Access This article is distributed under the terms of the Creative Commons Attribution 4.0 International License (http://crea tivecommons.org/licenses/by/4.0/), which permits unrestricted use, distribution, and reproduction in any medium, provided you give appropriate credit to the original author(s) and the source, provide a link to the Creative Commons license, and indicate if changes were made.

\section{References}

Carlson TN, Ripley DA (1997) On the relation between NDVI, fractional vegetation cover and leaf area Index. Remote Sens Environ 62(3):241-252

Liu XF, Yanu Y, Ren ZY, Lin ZH (2013) Changes of vegetation coverage in the loess plateau in 2000-2009. J Desert Res 33(4): 1244-1249

Yang LN, Niu SW, MA LG, Li YX (2010) Characteristics and cause analysis of land use/cover change in Dunhuang City. Chin J Ecol 29(4):766-775

Ye GX, Li WQ, Tian Y (2009) Dynamic changes of vegetation cover in typical oasis of arid areas based on NDVI-a case study in Cele Oasis. J Arid Land Resour Environ 23(9):128-133

Zhang H, Qu JJ, Zhang KC (2015) Vegetation cover information extraction technology for Dunhuang oasis based on remote sensing images. J Desert Res 35(2):229-234

Zhang ZP, Li ZY, Li QH, Hao RX (2017) Dynamic analysis on the vegetation coverage changes of Minqin Oasis based on GF-1 from 2013 to 2015. J Southwest For Univ 37(2):163-170 